\title{
Erratum to: Symptom attributions in patients with colorectal cancer
}

\author{
Line Flytkjær Jensen ${ }^{1,2^{*}+}$, Line Hvidberg ${ }^{1,2+}{ }^{\text {, Anette Fischer Pedersen }}{ }^{1}$ and Peter Vedsted ${ }^{1}$
}

Unfortunately, the original version of this article [1] contained an error. The original article was published containing the following paragraph in the abstract "Patients who experienced 'blood in stool' as the most important symptom were more likely to attribute this to cancer $(\mathrm{PR}$ ad 1.90, $95 \%$ CI 1.43-2.54) and benign somatic causes ( $\mathrm{PR}$ ad 1.33, $95 \%$ CI 1.02-1.72), such as haemorrhoids, compared to patients who did not perceive this symptom as the most important. Socio-demographic characteristics were also associated with symptom attribution. Patients with higher educational levels were less likely to attribute their most important symptom to psychological causes (PR ad 0.57, $95 \%$ CI 0.35-0.94) than patients with lower educational levels. Patients with rectal cancer attributed their most important symptom to a benign somatic cause more often than patients with colon cancer ( $\mathrm{PR}$ ad 1.39, 95 \% CI 1.07-1.80)."

This should have read "Patients who experienced 'blood in stool' as the most important symptom were more likely to attribute this to cancer (PR ad 1.94, $95 \%$ CI 1.46-2.58) and benign somatic causes ( $P R$ ad 1.36 , $95 \%$ CI 1.05-1.76), such as haemorrhoids, compared to patients who did not perceive this symptom as the most important. Socio-demographic characteristics were also associated with symptom attribution. Patients with higher educational levels were less likely to attribute their most important symptom to psychological causes ( $P R_{\text {ad }} 0.57,95 \%$ CI 0.34-0.96) than patients with lower educational levels. Patients with rectal cancer attributed their most important symptom to a benign somatic cause more often than patients with colon cancer ( $P R$ ad 1.34, 95 \% CI 1.02-1.77)."

This has now been corrected in the original article.

\footnotetext{
*Correspondence: line.jensen@ph.au.dk

${ }^{\dagger}$ Equal contributors

${ }^{1}$ Research Centre for Cancer Diagnosis in Primary Care (CaP), Research Unit for General Practice, Department of Public Health, Aarhus University, Bartholins Allé 2, Aarhus C 8000, Denmark

${ }^{2}$ Section for General Medical Practice, Department of Public Health, Aarhus University, Bartholins Allé 2, Aarhus C 8000, Denmark
}

Received: 7 September 2015 Accepted: 8 September 2015 Published online: 05 October 2015

\section{Reference}

1. Family Practice 2015, 16:115 doi: 10.1186/s12875-015-0315-9.

\section{Submit your next manuscript to BioMed Central and take full advantage of:}

- Convenient online submission

- Thorough peer review

- No space constraints or color figure charges

- Immediate publication on acceptance

- Inclusion in PubMed, CAS, Scopus and Google Scholar

- Research which is freely available for redistribution

Submit your manuscript at www.biomedcentral.com/submit 